\title{
Casimir effect around a screw dislocation
}

\author{
Ivan Pontual and Fernando Moraes \\ Departamento de Física \\ Universidade Federal de Pernambuco \\ 50670-901 Recife, PE, Brazil
}

\begin{abstract}
In this work, it is shown that a non-zero vacuum energy density (the Casimir energy) for a scalar field appears in a continuous elastic solid due to the presence of a topological defect, the screw dislocation. An exact expression is obtained for this energy density in terms of the Burgers vector describing the defect, for zero and finite temperature.
\end{abstract}




\section{Introduction}

The importance of the Casimir effect [1, 2, 3] in nowadays physics can hardly be overemphasized. It lies at the core of the modern definition of vacuum energy of quantum fields. Ideally, these fields are placed in an unbounded, infinite Minkowski spacetime. In practice, however, they may undergo interactions, which can be viewed as suitable boundary conditions on them, or they may be defined in a background spacetime of non-trivial topology (presence of defects and boundaries) or geometry (curvature and/or torsion). This causes variations in the zero-point energy of the quantum fields, and the difference between the modified energy and the Minkowskian one is the so-called Casimir energy (see, e.g., Ref. [2] for examples.).

The Casimir effect in the presence of conical topologies has been extensively studied in the context of cosmic strings [4, 5, 6, 7], but the applications in condensed matter systems have remained quite unexplored. Topological defects in solids (disclinations and dislocations) offer a natural setting for vacuum polarization to occur, as pointed out in a recent paper by one of us (F.M.) [8]. It was studied therein the Casimir effect in the presence of a disclination. The natural step further is to extend such results to the dislocation case, as these are more common in a solid [9].

In this work we calculate the Casimir energy for a scalar field in the presence of a screw dislocation, which is done by studying this field in a spacetime endowed with a non-Euclidean metric simulating the defect [10]. The reason as to why we deal with this defect is that it proved to be the most tractable one after the disclination case, and also because it is related to the still unsolved problem of the Casimir effect in the presence of a rotating cosmic string [5, 11], which brings naturally cosmology and solid state physics together formally, for mutual benefit. The scalar field is the simplest field and can be immediately generalized to the electromagnetic and phonon fields cases by just decomposing these along their polarization axis. Also, in Ref. [8] it was argued that fermionic fields (electrons in this case) should have a much smaller associated Casimir effect than the electromagnetic field due to an exponential damping related to the electronic mass.

This paper is organized as follows: in Sec.2 we review briefly the $\zeta$ function regularization technique as introduced by Hawking in Ref. [12]. We then use it in Sec. 3 to obtain an expression for the contribution of the vacuum energy due to the defect. In Sec. 4 we derive an asymptotic expres- 
sion for large distances, useful for practical purposes. In Sec. 5 we present our concluding remarks. In Appendix A we give a brief definition of the heat kernel of an operator and its connection with the generalized $\zeta$-function, for the benefit of the reader unfamiliar with these concepts. The heat kernel for the screw dislocation is obtained in Appendix B. Throughout the paper we use units in which $c=\hbar=k_{B}=1$.

\section{$2 \quad \zeta$-function regularization}

The $\zeta$-function technique [12, 13] is a powerful tool to calculate the Casimir energy. Among its advantages we have that the effects due to temperature may be implemented in a very straightforward manner, via the Euclidean time formalism 114. As we have a spatial defect, we shall be concerned with an ultrastatic spacetime manifold $\mathcal{M}$, i.e., $\mathcal{M}=R \times \Sigma$, where $\Sigma$ is $R^{3}$ modified by the defect. We may perform a Wick rotation of the time coordinate $x^{0}$ to the imaginary time $\tau=i x^{0}$, and impose that the scalar field $\varphi$ be periodic in imaginary time with period $\beta=\frac{1}{T}$, the inverse temperature. In this context, the partition function for the scalar field with mass $m$ at temperature $T$ is defined as (our notation and outlook follow [15):

$$
Z_{\beta}=\int_{\varphi(0, x)=\varphi(\beta, x)} \mathcal{D} \varphi \exp \left(-\frac{1}{2} \int_{\mathcal{M}} \varphi L_{4} \varphi d^{4} x\right)=\exp \left[\frac{1}{2} \zeta_{\beta}^{\prime}\left(0 \mid \frac{L_{4}}{\mu^{2}}\right)\right],
$$

where $\mu$ is an arbitrary renormalization parameter from the path integral measure, $L_{4}=-\Delta_{L B}^{(4)}+m^{2}$, and we represented by $\zeta_{\beta}^{\prime}\left(0 \mid \frac{L}{\mu^{2}}\right)$ the derivative at zero of the (generalized) $\zeta$-function of the operator $L_{4}$ (see Appendix A). After a Wick rotation the topology of $\mathcal{M}$ becomes $S^{1} \times \Sigma$, and $\Delta_{L B}^{(4)}$ stands for the four dimensional Laplace-Beltrami operator for the (Euclidianized) metric on $\mathcal{M}$ :

$$
\Delta_{L B}^{(4)}=\frac{1}{\sqrt{g}} \partial_{\mu}\left[\sqrt{g} g^{\mu \nu} \partial_{\nu}\right],
$$

where $g_{\mu \nu}$ is the metric tensor on $\mathcal{M}=S^{1} \times \Sigma$, and $g$ is its determinant. For Wick-rotated ultrastatic manifolds, one has that the LB operator can be decomposed as $\Delta_{L B}^{(4)}=-\partial_{\tau}-\Delta_{L B}^{(3)}$, and now $\Delta_{L B}^{(3)}$ is the LB operator for the metric on $\Sigma$ (spatial part of the metric on $\mathcal{M})$.

Eq. (1) provides all relevant regularized physical quantities via the usual formulas of thermodynamics, with one difference from the ordinary partition 
function: the presence of a vacuum term, which gives the zero-temperature contribution. This can be isolated in the limit $\beta \rightarrow \infty$. By denoting $L_{3}=$ $-\Delta_{L B}^{(3)}+m^{2}$, one can use the relation between the heat kernel of an operator and its $\zeta$-function (Appendix A) and some algebraic manipulations to obtain [16] :

$$
\begin{aligned}
\zeta\left(s \mid \frac{L_{4}}{\mu^{2}}\right)= & \frac{\mu \beta \Gamma(s-1 / 2)}{\sqrt{4 \pi} \Gamma(s)} \zeta\left(s-1 / 2 \mid \frac{L_{3}}{\mu^{2}}\right)+\frac{\mu \beta}{\sqrt{\pi} \Gamma(s)} \sum_{n=1}^{\infty} \int_{0}^{\infty} t^{s-1 / 2} \times \\
& e^{-(n \mu \beta)^{2} / 4 t} T r e^{-t L_{3} / \mu^{2}} d t,
\end{aligned}
$$

from which it is easily deduced $[15,16]$, that

$$
\ln Z_{\beta}=-\frac{\beta}{2} \zeta\left(-1 / 2 \mid L_{3}\right)+\frac{\beta}{\sqrt{4 \pi}} \sum_{n=1}^{\infty} \int_{0}^{\infty} t^{-3 / 2} e^{-(n \beta)^{2} / 4 t} \operatorname{Tr} e^{-t L_{3}} d t
$$

in the case one has scale independence of the Casimir energy 円. In Eqs. (3) and (4), $\operatorname{Tr} e^{-t L_{3}}$ stands for the trace of the heat kernel of $L_{3}$ (Appendix A). We shall be interested in the free energy of the field $F=-\ln Z_{\beta} / \beta$, which immediately yields

$$
F=\frac{1}{2} \zeta\left(-1 / 2 \mid L_{3}\right)-\frac{1}{\sqrt{4 \pi}} \sum_{n=1}^{\infty} \int_{0}^{\infty} t^{-3 / 2} e^{-(n \beta)^{2} / 4 t} \operatorname{Tr} e^{-t L_{3}} d t
$$

This last equation shows clearly the splitting between the $T=0$ term and the contribution from thermal excitations, the latter being identified with the ordinary thermodynamical free energy. One has hereby a conceptually simple method to calculate the Casimir energy, just by obtaining the heat kernel of $L_{3}$ and its $\zeta$-function. We proceed to do this in next section, for the explicit case of a screw dislocation.

\section{Casimir energy around a screw dislocation}

In this section we basically use a variant of Eq. (5) to obtain an expression for the Casimir energy. We follow the theory of defects/three-dimensional

\footnotetext{
${ }^{1}$ The Casimir energy may depend on the scale $\mu$ and in this case the expression is more complicated. In Ref. [15] it is displayed the general formula. In our case, and in the majority of the physically relevant systems, one has scale independence, and therefore we omit further comments on this. For a discussion of these issues, see Ref. [23].
} 
gravity of Katanaev-Volovich [17] to model a screw dislocation by endowing the elastic infinite continuum representing the solid with a metric given, in cylindrical coordinates, by [10]:

$$
d s^{2}=d r^{2}+r^{2} d \phi^{2}+(d z+\kappa d \phi)^{2},
$$

where $0 \leq \phi \leq 2 \pi$. This metric represents an infinite defect along the z-axis, resulting from "cutting" the space along the semiplane $\phi=0, r \geq 0$, and "sliding " it along the $\mathrm{z}$ axis by an amount $b$, the modulus of the Burgers vector. This process gives a helix-like topology to the space, the step of which is $\kappa \equiv \frac{b}{2 \pi}$. In the case $b=0$ we obviously recover the flat Euclidean metric, describing the medium in the absence of dislocations. The metric in Eq. (6) is locally flat, as can be easily seen by performing the coordinate transformation $Z^{\prime} \equiv z+\kappa \phi$ :

$$
d s^{2}=d r^{2}+r^{2} d \phi^{2}+d Z^{\prime 2}
$$

which is flat everywhere except at $r=0$, where it has a $\delta$-function singularity in the torsion [10]. One may infer the helix-like behavior by noting that the periodicity of $\phi$ induces the identification:

$$
\left(t, r, \phi, Z^{\prime}\right) \sim\left(t, r, \phi+2 \pi, Z^{\prime}+2 \pi \kappa\right) .
$$

As we mentioned before, our interest is in deriving the heat kernel of the operator $L_{3}$ associated with this metric. This is done in Appendix B, and the result is:

$$
\begin{aligned}
K\left(\vec{r}, \vec{r}^{\prime}, t\right)= & \frac{e^{-m^{2} t} e^{-\frac{\left(r^{2}+r^{\prime 2}\right)}{4 t}}}{2(2 \pi)^{2} t} \sum_{n=-\infty}^{\infty} e^{-i n\left(\phi-\phi^{\prime}\right)} \int_{-\infty}^{\infty} e^{-\nu^{2} t} e^{i \nu\left(z-z^{\prime}\right)} \times \\
& I_{|n+\kappa \nu|}\left(\frac{r r^{\prime}}{2 t}\right) d \nu
\end{aligned}
$$

where $I_{p}(z)$ is the modified Bessel function of the first kind. If we consider $\vec{r}=\vec{r}^{\prime}$, Eq.(9) becomes:

$$
K(\vec{r}, \vec{r}, t)=\frac{e^{-m^{2} t} e^{-\frac{r^{2}}{2 t}}}{2(2 \pi)^{2} t} \sum_{n=-\infty}^{\infty} \int_{-\infty}^{\infty} e^{-\nu^{2} t} I_{|n+\kappa \nu|}\left(\frac{r^{2}}{2 t}\right) .
$$


The so-called local $\zeta$-function, which plugged into Eq. (5) gives the free energy density (see Appendix A), is obtained by setting $\vec{r}=\vec{r}^{\prime}$ in Eq. (9) and applying a Mellin transform [18]:

$$
\zeta(s, \vec{r}) \equiv \frac{1}{\Gamma(s)} \int_{0}^{\infty} t^{s-1} K(\vec{r}, \vec{r}, t) d t .
$$

Before performing the Mellin transformation, however, we shall work Eq. (10) out to reshape it into a more convenient form. Let us consider the well known integral representation [18]:

$$
I_{\nu}(z)=\frac{1}{2 \pi} \int_{-\pi}^{\pi} e^{z \cos \theta} \cos \nu \theta d \theta-\frac{\sin \nu \pi}{\pi} \int_{0}^{\infty} e^{-z \cosh x-\nu x} d x,
$$

valid for $R e z>0$ and $R e \nu \geq 0$. We substitute it into Eq. (10) to obtain

$$
\begin{aligned}
K(\vec{r}, \vec{r}, t)= & \frac{e^{-m^{2} t} e^{-\frac{r^{2}}{2 t}}}{2(2 \pi)^{2} t} \sum_{n=-\infty}^{\infty}\left[\frac{1}{2 \pi} \int_{-\pi}^{\pi} d \theta e^{\frac{r^{2}}{2 t} \cos \theta} \int_{-\infty}^{\infty} d \nu e^{-\nu^{2} t} \times\right. \\
& \cos [(n+\kappa \nu) \theta]-\frac{1}{\pi} \int_{-\infty}^{\infty} d \nu e^{-\nu^{2} t} \sin |n+\kappa \nu| \times \\
& \left.\int_{0}^{\infty} d x e^{-\frac{r^{2}}{2 t} \cosh x} e^{-|n+\kappa \nu| x}\right] .
\end{aligned}
$$

We shall deal separately with the integrals. We have that [18]:

$$
\int_{-\infty}^{\infty} d \nu e^{-\nu^{2} t} \cos [(n+\kappa \nu) \theta]=\sqrt{\frac{\pi}{t}} e^{-\frac{\kappa^{2} \theta^{2}}{4 t}} \cos (n \theta) .
$$

We use here the Poisson summation formula [2]:

$$
\sum_{n=-\infty}^{\infty} F(n)=2 \pi \sum_{n=-\infty}^{\infty} c(2 \pi n)
$$

where

$$
c(\alpha)=\frac{1}{2 \pi} \int_{-\infty}^{\infty} d x e^{i \alpha x} F(x) .
$$

This easily gives the well-known formula:

$$
\sum_{n=-\infty}^{\infty} \delta(x-n)=\sum_{n=-\infty}^{\infty} e^{i 2 \pi n x}
$$


which provides the following representation:

$$
\sum_{n=-\infty}^{\infty} \cos (n \theta)=\frac{1}{2}\left[\sum_{n=-\infty}^{\infty} \delta\left(\frac{\theta}{2 \pi}-n\right)+\sum_{n=-\infty}^{\infty} \delta\left(\frac{\theta}{2 \pi}+n\right)\right] .
$$

We recall that $\delta(\lambda x)=\lambda^{-1} \delta(x)$, and upon substitution of Eq.(14) into Eq.(13), and then substituting Eq. (18) into Eq. (13), we obtain:

$$
\begin{aligned}
K(\vec{r}, \vec{r}, t)= & \frac{e^{-m^{2} t}}{(4 \pi t)^{3 / 2}}-\frac{e^{-m^{2} t} e^{-\frac{r^{2}}{2 t}}}{8 \pi^{3} t} \sum_{n=-\infty}^{\infty} \int_{-\infty}^{\infty} d \nu e^{-\nu^{2} t} \times \\
& \sin \pi|n+\kappa \nu| \int_{0}^{\infty} d x e^{-\frac{r^{2}}{2 t} \cosh x} e^{-|n+\kappa \nu| x} .
\end{aligned}
$$

The next step is to calculate the local $\zeta$-function (see Appendix A):

$$
\zeta(\vec{r}, s)=\frac{1}{\Gamma(s)} \int_{0}^{\infty} t^{s-1} K(\vec{r}, \vec{r}, t) d t
$$

which by means of a simple representation of the modified Bessel function of the second kind,

$$
2\left(\frac{\alpha}{\alpha^{\prime}}\right)^{\nu / 2} K_{\nu}\left(2 \sqrt{\alpha \alpha^{\prime}}\right)=\int_{0}^{\infty} d t t^{\nu-1} \exp \left(-\frac{\alpha}{t}-\alpha^{\prime} t\right),
$$

may be written as:

$$
\begin{aligned}
\zeta(\vec{r}, s)= & \frac{\Gamma(s-3 / 2) m^{-2 s+3}}{(4 \pi)^{3 / 2} \Gamma(s)}-\frac{1}{4 \pi^{3} \Gamma(s)} \int_{-\infty}^{\infty} d \nu \int_{0}^{\infty} d x F_{b}(\nu, x) \times \\
& \left(\frac{r \cosh \left(\frac{x}{2}\right)}{\sqrt{\nu^{2}+m^{2}}}\right)^{s-1} K_{s-1}\left(2 r \cosh \left(\frac{x}{2}\right) \sqrt{\nu^{2}+m^{2}}\right)
\end{aligned}
$$

where we have defined

$$
F_{b}(\nu, x)=\sum_{n=-\infty}^{\infty} \sin \pi|n+\kappa \nu| e^{-|n+\kappa \nu| x} .
$$

We may notice that for $\kappa=0$ this last summation vanishes. Therefore, in the absence of the defect $(b=0)$, we see that only the first term on the right 
hand side of Eq.(22) remains. By substituting the Euclidean heat kernel, Eq.(B.7) of Appendix B, into Eq.(11), we see that this term corresponds to the free space $\zeta$-function. The Casimir energy is renormalized with respect to the flat, infinite Euclidean space, and we shall accordingly disregard the Euclidean term and thus obtain the defect $\zeta$-function:

$$
\begin{aligned}
\zeta_{b}(\vec{r}, s)= & -\frac{1}{4 \pi^{3} \Gamma(s)} \int_{-\infty}^{\infty} d \nu \int_{0}^{\infty} d x F_{b}(\nu, x) \times \\
& \times\left(\frac{r \cosh \left(\frac{x}{2}\right)}{\sqrt{\nu^{2}+m^{2}}}\right)^{s-1} K_{s-1}\left(2 r \cosh \left(\frac{x}{2}\right) \sqrt{\nu^{2}+m^{2}}\right) .
\end{aligned}
$$

As it stands, we have the Casimir energy density at zero temperature: it is simply $E=\frac{1}{2} \zeta_{b}(\vec{r}, s)$. We may get the full energy, valid for any temperature by considering Eq.(A.11) of the Appendix A. It is clear that our problem is to calculate the integral :

$$
I_{p}=\int_{0}^{\infty} t^{-3 / 2} K(\vec{r}, \vec{r}, t) e^{-\frac{(p \beta)^{2}}{4 t}} d t
$$

for each natural number $p$. The procedure is entirely analogous to the former calculation, and it yields:

$$
\begin{aligned}
I_{p}= & -\frac{1}{4 \pi^{3}} \int_{-\infty}^{\infty} d \nu \int_{0}^{\infty} d x F_{b}(\nu, x)\left[\sqrt{\frac{r^{2} \cosh ^{2}\left(\frac{x}{2}\right)+\frac{(p \beta)^{2}}{4}}{\nu^{2}+m^{2}}}\right]^{-3 / 2} \times \\
& K_{-3 / 2}\left(2 \sqrt{r^{2} \cosh ^{2}\left(\frac{x}{2}\right)+\frac{(p \beta)^{2}}{4}} \sqrt{\nu^{2}+m^{2}}\right)
\end{aligned}
$$

Then, we finally have the free energy density for a massive scalar field in the presence of a screw dislocation at any temperature:

$$
\begin{aligned}
\mathcal{F}= & \frac{1}{2} \zeta_{b}(\vec{r},-1 / 2)+\frac{1}{8 \pi^{3}} \sum_{p=1}^{\infty} \int_{-\infty}^{\infty} d \nu \int_{0}^{\infty} d x F_{b}(\nu, x)\left[\sqrt{\frac{r^{2} \cosh ^{2}\left(\frac{x}{2}\right)+\frac{(p \beta)^{2}}{4}}{\nu^{2}+m^{2}}}\right]^{-3 / 2} \times \\
& K_{-3 / 2}\left(2 \sqrt{r^{2} \cosh ^{2}\left(\frac{x}{2}\right)+\frac{(p \beta)^{2}}{4}} \sqrt{\nu^{2}+m^{2}}\right) .
\end{aligned}
$$


The massless case may also be otained by directly setting $m=0$ in this equation. The value $\zeta_{b}(\vec{r},-1 / 2)$ should be understood in the following way: one assumes that $s$ is large enough to make the integrals in Eq.(22) converge, performs the integration in terms of $s$ and afterwards one analitically continues the resulting expression to $s=-1 / 2$. This cannot be done explicitly in our case due to the complexity of the expressions. Thus we have derived an exact expression for the Casimir energy, but because it is a complicated one, in the next section we shall derive an asymtotic formula for the simplest particular case, namely the massless field at zero temperature.

\section{Asymptotic expression}

Our problem here amounts to see what our formulas become if we take $\frac{r r^{\prime}}{2 t} \rightarrow$ $\infty$. From the physical standpoint, one expects that the qualitative features of the energy functional are preserved at large distances, since the defect is axially symmetric and infinite. On the other hand, our considering the solid as a continuum medium is an approximation valid for large distances from the defect.

Let us now consider Eq.(10) for the heat kernel. We set $m=0$ and and take the following asymptotic expression for the modified Bessel function for $|w| \rightarrow \infty$ [18]:

$$
I_{|\lambda|}(w) \approx(2 \pi w)^{-1 / 2} \exp \left(w-\frac{1}{2 w}\left(\lambda^{2}-1 / 4\right)\right) .
$$

Now, we just set $w=\frac{r^{2}}{2 t}$ and $\lambda=n+\kappa \nu$, and substitute Eq.(28) into Eq.(10).The result is:

$$
K(\vec{r}, \vec{r}, t)=\frac{e^{\frac{t}{4 r^{2}}}}{8 \pi^{2} \sqrt{\pi t}} \sum_{n=-\infty}^{\infty} e^{-\frac{n^{2} t}{r^{2}}} \int_{-\infty}^{\infty} d \nu e^{-t\left(1+\frac{\kappa^{2}}{r^{2}}\right)\left(\nu^{2}+\frac{2 \kappa \nu n}{r^{2}\left(1+\frac{\kappa^{2}}{r^{2}}\right)}\right)} .
$$

We complete the square in the last exponencial and, defining a new variable $u \equiv \nu+\frac{\kappa n}{r^{2}\left(1+\frac{\kappa^{2}}{r^{2}}\right)}$, integrate with respect to $u$ to obtain:

$$
K(\vec{r}, \vec{r}, t)=\frac{e^{\frac{t}{4 r^{2}}}}{8 \pi^{2} t r}\left(1+\frac{\kappa^{2}}{r^{2}}\right)^{-1 / 2} \sum_{n=-\infty}^{\infty} e^{-\frac{t}{r^{2}} n^{2}} e^{t \frac{n^{2} \kappa^{2}}{r^{2}\left(1+\frac{\kappa^{2}}{r^{2}}\right)}} .
$$


Thus, we simply expand $e^{\frac{t}{4 r^{2}}} \approx 1+\frac{t}{4 r^{2}}$ and apply the Mellin transform, which yields the local zeta function for the massless field:

$$
\begin{aligned}
\zeta_{(m=0)}(s, \vec{r})= & \frac{\Gamma(s-1)}{4 \pi^{2} \Gamma(s)} r^{2 s-3}\left(1+\frac{\kappa^{2}}{r^{2}}\right)^{s-3 / 2} \zeta_{R}(2 s-2)+ \\
& \frac{1}{16 \pi^{2} \Gamma(s)} r^{2 s-3}\left(1+\frac{\kappa^{2}}{r^{2}}\right)^{s-3 / 2} \zeta_{R}(2 s),
\end{aligned}
$$

where we have substituted $\sum_{n=1}^{\infty} n^{-z} \equiv \zeta_{R}(z)$, the Riemann zeta function [18]. Now we put $s=-1 / 2$ and pick the values of the Riemann zeta function and gamma function in any standard table of special functions, e.g., Ref. [18], then we finally have the simple expression:

$$
\zeta_{(m=0)}(-1 / 2, \vec{r})=-\frac{1}{720 \pi^{2}}\left(1+\frac{\kappa^{2}}{r^{2}}\right)^{-2} \frac{1}{r^{4}}-\frac{1}{480 \pi^{2}}\left(1+\frac{\kappa^{2}}{r^{2}}\right)^{-1} \frac{1}{r^{4}} .
$$

We have seen that we must subtract the Euclidean contribution in order to obtain the renormalized Casimir energy density:

$$
\mathcal{E}_{\text {ren. }}=\frac{1}{2}\left[\zeta_{(m=0)}(-1 / 2, \vec{r})-\left.\zeta_{(m=0)}(-1 / 2, \vec{r})\right|_{\kappa=0}\right] .
$$

From this expression we obtain, for instance, the lowest order term of an expansion in powers of $\frac{\kappa^{2}}{r^{2}}$ :

$$
\mathcal{E}_{\text {ren. }}=\frac{7}{2880 \pi^{2}} \frac{\kappa^{2}}{r^{6}}+\mathcal{O}\left(\frac{\kappa^{4}}{r^{8}}\right) .
$$

Note that in the units we use here the energy has units of (lenght) ${ }^{-1}$ That the sign is positive should already be expected, since at $T=0$ the addition of the defect to the background increases the energy of the field,i.e., the favoured configuration is $b=0$, the flat space without defects. Only even powers of $\kappa$ occur, since it is immaterial from an energetic viewpoint whether the "helix" is right-handed ( $b$ positive) or left-handed ( $b$ negative).

\section{Concluding Remarks}

In the present work we give an example of the importance of quantum field theoretical effects in solids with defects. Albeit very simplified, our model 
for the solid (infinite, non-magnetic and with only one defect), gives already a non-zero Casimir energy, which can play a role in various phenomena in solid state physics, for instance, contributing to the specific heat of solids.

It would be interesting to investigate an extension of this model to magnetic materials. In the continuous approximation of the Ising model, for instance, this amounts to add a term proportional to $\varphi^{4}$ [21] to the argument of the first exponential in Eq.(1). This model would no longer yield an exact result but could be handled perturbatively.

Another line of interest is to consider classical effects of the defect. Specifically, it is known 22] that a classical point charge in the presence of a disclination suffers a self force induced by the defect. An identical effect would be expected in the dislocation case, and possibly give rise to bound states. We are presently tackling this problem.

\section{Appendix A: $\zeta$-function and the heat kernel operator}

The $\zeta$-function technique as used in this paper was sistematically applied for the first time in Ref. [13], but Hawking [12] shaped it into its present form. We briefly introduce the subject in this Appendix. For a more detailed account we suggest Ref. [12 and the comprehensive (and very complete) book by Elizalde et al. [19].

One starts with the description of a quantum physical system by a Hamiltonian $\hat{H}$, with adequate boundary conditions and, possibly, non-trival background metric and topology. Mathematically, this reduces to an elliptic, self-adjoint, second order differential operator, with correspondent boundary conditions. Generally, it is not possible to calculate its spectrum directly, but we can still define, for such a kind of operator, the (generalized) zeta function of $\hat{H}$, given by:

$$
\zeta_{\hat{H}}(s) \equiv \operatorname{Tr} \exp (-s \ln \hat{H}), s \in C .
$$

In the case where we have a known spectrum, $\left\{\lambda_{n}\right\}$ ( for simplicity, we assume in what follows that the spectrum is discrete), the operator $e^{-s \ln \hat{H}}$ may be diagonalized, becoming an infinite "matrix", with entries $e^{-s \ln \lambda_{n}}$, and therefore the trace in Eq.(A.1) becomes:

$$
\zeta_{\hat{H}}(s) \equiv \sum_{n} \lambda_{n}^{-s}
$$


Of course, if one has a zero eigenvalue, it is dealt with separately. If $\lambda_{n}=n$, where $n$ is a positive integer, and the summation runs over the positive integers, one has just the well known Riemann zeta function [18], and hence its name. Even if the spectrum is not known, one can still obtain information about the $\zeta$-function by the so-called heat equation of the operator $\hat{H}$ :

$$
\frac{\partial}{\partial t} F(x, y, t)+\hat{H} F(x, y, t)=0
$$

where $x$ and $y$ are spacetime points, and $t$ is a parameter not necessarily equal to the coordinate time. We assume that $\hat{H}$ acts with respect to $x$ only, and that we have the initial condition

$$
F(x, y, 0) \equiv \delta(x-y) .
$$

Of course, Eq.(A.3) reduces to the ordinary heat equation if we have $\hat{H}=$ $-\Delta$, the ordinary Laplacian. The function $F(x, y, t)$ (actually a distribution) obeying Eq.(A.3) with condition as Eq.(A.4) is called the heat kernel of the operator $\hat{H}$.

Now let $\left\{\varphi_{n}(x)\right\}$ be a complete set of eigenfunctions of $\hat{H}$, orthonormalized by the standard inner product:

$$
\int \varphi_{n}(x) \varphi_{m}(x) \sqrt{g} d x=\delta_{m n},
$$

where $g$ is the determinant of the metric tensor on the spacetime, or region of spacetime under consideration. Of course, they have associated eingenvalues:

$$
\hat{H} \varphi_{n}=\lambda_{n} \varphi_{n} \text {. }
$$

In terms of these eigenvalues and eigenfunctions the heat kernel may be written as:

$$
F(x, y, t)=\sum_{n} \exp \left(-\lambda_{n} t\right) \varphi_{n}(x) \varphi_{n}(y) .
$$

The trace of the heat kernel is defined as:

$$
\operatorname{Tr} \exp (-t \hat{H}) \equiv \int F(x, x, t) \sqrt{g} d x=\sum_{n} \exp \left(-\lambda_{n} t\right)
$$

Note that $F(x, y, t)$ is the "matrix "element of the operator $\exp (-t \hat{H})$. The link with the $\zeta$-function is established via a Mellin transform [12]:

$$
\zeta_{\hat{H}}(s)=\frac{1}{\Gamma(s)} \int_{0}^{\infty} t^{s-1} \operatorname{Tr} \exp (-t \hat{H}) d t
$$


One may also define the local $\zeta$-function :

$$
\zeta_{\hat{H}}(x, s)=\frac{1}{\Gamma(s)} \int_{0}^{\infty} t^{s-1} F(x, x, t) d t
$$

in terms of which one may adapt Eq.(5) to obtain the free energy density:

$$
\mathcal{F}=\frac{1}{2} \zeta_{\hat{H}}(x,-1 / 2)-\frac{1}{\sqrt{4 \pi}} \sum_{n=1}^{\infty} \int_{0}^{\infty} t^{-3 / 2} e^{-(n \beta)^{2} / 4 t} F(x, x, t) d t .
$$

The convenience of taking local quantities is avoiding an infinite global $\zeta$-function due to an infinite spacetime.

\section{Appendix B: the heat kernel for the screw dislocation}

In Section III, we displayed the heat kernel for the operator $L_{3}=-\Delta_{L B}+m^{2}$ for the metric describing the screw dislocation, Eq.(6). We also showed in Appendix A that the problem of obtaining the heat kernel actually boils down to knowing the eingenvalues and (orthonormalized) eigenfunctions of the respective operator, and using Eq.(A.7). The eigenvalue equation can be written for the screw dislocation metric as:

$$
\left[-\frac{1}{r} \frac{\partial}{\partial r}\left(r \frac{\partial}{\partial r}\right)-\frac{1}{r^{2}}\left(\frac{\partial}{\partial \phi}-\kappa \frac{\partial}{\partial z}\right)^{2}-\frac{\partial^{2}}{\partial z^{2}}+m^{2}\right] \varphi(\vec{r})=\lambda \varphi(\vec{r}) .
$$

A complete set of orthonormal solutions is easily seen to be:

$$
\varphi_{n, \nu, k}(r, \phi, z)=\frac{1}{2 \pi} J_{|n+\kappa \nu|}(k r) e^{-i n \phi} e^{i \nu z}
$$

and its complex conjugate. $\lambda=k^{2}+\nu^{2}+m^{2}$, where $k$ is a positive real number, $\nu$ is a real number, and $n$ is an integer. $J_{|n+\kappa \nu|}(k r)$ is the regular Bessel function. Application of Eq.(A.7) gives (the measure of integration for $k$ is $k d k)$ :

$$
\begin{aligned}
K\left(\vec{r}, \vec{r}^{\prime}, t\right)= & \frac{e^{-m^{2} t}}{(2 \pi)^{2}} \sum_{n=-\infty}^{\infty} \int_{-\infty}^{\infty} d \nu e^{-\nu^{2} t} \int_{0}^{\infty} d k k e^{-k^{2} t} e^{-i n\left(\phi-\phi^{\prime}\right)} e^{i \nu\left(z-z^{\prime}\right)} \times \\
& J_{|n+\kappa \nu|}(k r) J_{|n+\kappa \nu|}\left(k r^{\prime}\right) .
\end{aligned}
$$


Using the relation 18:

$$
\int_{0}^{\infty} e^{-a^{2} x^{2}} J_{p}(\alpha x) J_{p}(\beta x) x d x=\frac{e^{-\frac{\left(\alpha^{2}+\beta 2\right)}{4 a^{2}}}}{2 a^{2}} I_{p}\left(\frac{\alpha \beta}{2 a^{2}}\right)
$$

we arrive at the form:

$K\left(\vec{r}, \vec{r}^{\prime}, t\right)=\frac{e^{-m^{2} t} e^{-\frac{\left(r^{2}+r^{\prime 2}\right)}{4 t}}}{2(2 \pi)^{2} t} \sum_{n=-\infty}^{\infty} e^{-i n\left(\phi-\phi^{\prime}\right)} \int_{-\infty}^{\infty} d \nu e^{-\nu^{2} t} e^{i \nu\left(z-z^{\prime}\right)} I_{|n+\kappa \nu|}\left(\frac{r r^{\prime}}{2 t}\right)$.

In order to show that it is consistent, we put $b=0$ to show it reduces to the Euclidean case. Using the identity [18]:

$$
e^{\frac{x}{2}\left(t+\frac{1}{t}\right)}=\sum_{n=-\infty}^{\infty} I_{n}(x) t^{n}
$$

we obtain:

$$
K_{b=0}\left(\vec{r}, \vec{r}^{\prime}, t\right)=\frac{e^{-m^{2} t} e^{-\frac{\left(\vec{r}-\vec{r}^{\prime}\right)^{2}}{4 t}}}{(4 \pi t)^{3 / 2}},
$$

which in fact is the Euclidean heat kernel [20].

\section{Acknowledgment}

This work was partially supported by CNPq.

\section{References}

[1] H.B. Casimir, Proc. Kon. Ned. Akad. Wet. 51, 793 (1948).

[2] G. Plunien, B. Müller and W. Greiner, Phys. Rep. 134 , 87 (1986).

[3] V.M. Mostepanenko and N.N. Trunov, Sov. Phys. Usp. 31, 965 (1988).

[4] A. Vilenkin, Phys. Rep. 121, 263 (1985).

[5] E.S. Moreira Jr., Nucl. Phys. B451, 365 (1995).

[6] B. Linet, Phys. Rev. D35, 536 (1987). 
[7] J.S. Dowker, Phys. Rev. D36, N. 10, 3095 (1987).

[8] F. Moraes, Phys. Lett. A 204, 399 (1995).

[9] C. Kittel, "Introduction to Solid State Physics", $4^{\text {th }}$ Ed., J. Willey \& Sons, N. York (1971).

[10] K.P. Tod, Class Quantum Grav. 11, 1331 (1994).

[11] G.E.A. Matsas, Phys. Rev. D42, 2927 (1990).

[12] S.W. Hawking, Commun. Math. Phys. 55, 133 (1977).

[13] J.S. Dowker and R. Critchley, Phys. Rev. D13, N.12, 3224 (1976).

[14] H. Kleinert, "Gauge Fields in Condensed Matter", Vols. I and II, World Scientific, Singapore, (1989).

[15] G. Cognola, K. Kirsten and L.Vanzo, Phys. Rev. D49, 1029 (1994).

[16] G. Cognola, L. Vanzo and S. Zerbini, J. Math. Phys. 33, 222 (1992).

[17] N.O. Katanaev and I.V. Volovich, Ann. of Phys. (N.Y.) 1, 216 (1992).

[18] I.S. Gradshteyn and I.M. Ryzhik, "Table of Integrals, Series and Products", Academic Press, N.York (1980).

[19] E.Elizalde, S.P. Odintsov, A. Romeo, A.A. Bytsenko and S. Zerbini, "Zeta Function Regularization Techniques with Applications", World Scientific, Singapore (1994).

[20] G. Cognola, Phys. Rev. D50,909 (1994).

[21] D.J. Amit, "Field Theory, the Renormalization Group, and Critical Phenomena", World Scientific, Singapore (1984).

[22] C. Furtado and F. Moraes, Phys. A188, 394 (1994).

[23] S.K. Blau, M. Visser and A.Wipf, Nucl. Phys. B310, 163 (1988). 